\title{
OFDM Synchronization Techniques for 802.11ac WLAN
}

\author{
Chandresh D. Parekha ${ }^{\mathrm{a}}$, Dr. Jayesh M. Patel ${ }^{\mathrm{b}}$ \\ ${ }^{a}$ Gujarat Technological University, Ahmedabad, Gujarat, India \\ ${ }^{b}$ Ganpat University, Kherva, Mehsana, Gujarat, India
}

Received: 30 November 2017; Accepted: 13 February 2018; Published: 08 July 2018

\begin{abstract}
It would be dire need of accurate and fast wireless communication in the present as well as future era. Few years ago, error free wireless link design was only challenge and it had opened a way of digital communication. Now speed of communication becomes vital and essential part of research in the field of wireless communication. In wired communication category, optical communication is a solution to achieve targeted data speed but wireless channel for serial communication is highly disturbed with the phenomena of multipath and frequency selective fading for a data rate over a specific limit. A new technology named Orthogonal Frequency Division Multiplexing (OFDM) is a perfect key to open this locking situation of limited data rate with optimum use of bandwidth in the multipath fading channel. Supporting high data rates and robust response against narrowband interferences, OFDM had penetrated itself in many broadband wireless services. There are many issues with OFDM too and one of them is synchronization between orthogonal frequency channels at receiver. It is mandatory condition in the use of OFDM technology to maintain orthogonality among subcarriers for error free communication. Timing and frequency synchronization errors are quite detrimental to this condition because these errors ultimately disturb the orthogonality which is perfectly set at transmitter. Synchronization errors need to be handled properly otherwise they create Inter-Symbol Interference (ISI) and Inter-Channel Interference (ICI) which after all resulted into the deterioration of Bit Error Rate (BER). This paper covers types of synchronization and its effects, algorithms to decrease it and analysis of the same.
\end{abstract}

Index Terms: Synchronization, OFDM, ISI, ICI, WLAN, CFO

(C) 2018 Published by MECS Publisher. Selection and/or peer review under responsibility of the Research Association of Modern Education and Computer Science

\section{Introduction}

It is reality that OFDM based digital modulation technique has set its inimitable position in the wireless communication whether it is a short distanced Wireless Local Area Network (WLAN) or a long distanced

* Corresponding author.

E-mail address: 
Digital Video Broadcasting (DVB). Next generation of wireless communication such as high speed internet, multimedia services and streaming videos would not be completed satisfactory without OFDM because it congregates high speed, good quality and better mobility requirements with reasonable and adequate spectrum efficiency. OFDM is basically a digital multi-carrier modulation scheme which supports elevated speed of communication by dividing a high speed data link into several low speed data links. Each low speed data link sends data using one of the several adjacent orthogonal subcarriers which are split from available broad channel. It is a magnificent invention in telecommunication field because without OFDM, it is impossible to design high speed wireless devices to be employed in frequency-selective fading channel. Currently, most of the broadband wireless communication systems are based on OFDM technology as it turns out to be a well-built technique with the benefit of spectral efficiency and simplicity in equalization.

OFDM concept is not new but it was first introduced in the year 1966 by chang. It did not become commercial and popular till 1990 because of hardware complexity, issues due to large number of subcarriers and cost. Later, with development of digital signal processing, OFDM had been accepted for most of the high speed communication systems such as HDSL, ADSL, VHDSL, HDTV, DVB and DAB [1]. It is also taken up in the IEEE standards $802.11 \mathrm{a} / \mathrm{g} / \mathrm{n} / \mathrm{ac}$ of wireless local area networks, HIPERLAN/2 [2] and wireless metropolitan area networks (WMAN/WiMax, IEEE 802.16 [3]. The third generation (3G) systems for mobile communication also use OFDM technology. The fourth generation (4G- LTE) systems [4] are supporting even more challenging physical layer requirements, such as video calling and streaming.

Though OFDM offers higher throughput with acceptable quality and affordable cost over the conventional single carrier digital modulation techniques, it is also facing regular issues of wireless transmission with few new challenges such as high peak-to-average power ratio (PAPR) and synchronization between transmitter and receiver. This is multicarrier communication in which time and frequency synchronization between closely spaced subcarriers in order to preserve orthogonality is very crucial for the precise detection of the signal at the receiver [6]. Timing and frequency synchronization errors together introduce additional interference to the OFDM systems which eventually degrade the performance. There are many algorithms and methods proposed to estimate and mitigate time and frequency synchronization errors which vary application to application because of variation in the data frame structure. While handling this critical issue in OFDM link of any application, it is advisable to retain overall cost and complexity of the system in predetermined limit so that it becomes commercially viable. Computationally complex synchronization algorithms will add complication in the calculation of Fast Fourier Transform (FFT) which has replaced bank of demodulators at the receiver.

The paper is aimed to talk about the synchronization issues for OFDM systems, some insights into the causes, effects and means of reducing them and at the last synchronization algorithms used in the 802.11ac standard of WLAN. Section 2 covers the basics of synchronization. Section 3 describes data frame structure for the IEEE 802.11ac standard employed for Wireless LAN. Section 4 analyzes the outcome of simulation and after that paper ends with some conclusions in the last section 5 .

\section{Synchronization}

Synchronization issue is one of the problems of multicarrier communication systems based on OFDM needs to be tackled for efficient result. Performance quality level of synchronization system is the main indicator of any communication system. Normally, Synchronization is being lost by timing and frequency offset at receiver. The way and method to handle both types of synchronization errors depend on application. Frequency and timing error estimation and correction decide the performance of OFDM system. In our paper [5], we have tried to provide essential information for primary and rudimentary study around synchronization issue in OFDM based communication links. Inter-carrier-interference (ICI) and its causes, effects and solutions are also discussed.

Basically, symbol and sampling clock synchronization together authenticate timing synchronization [7], [8]. Symbol synchronization error occurs due to spreading of OFDM symbols in frequency selective wireless channel which ultimately creates error in the detection of the starting of the symbol. This is called intersymbol-interference (ISI). ISI also occurs due to sampling clock offset because it generates drifting of the 
OFDM symbol block away from the receiver Fast Fourier Transform (FFT) window. These two causes of ISI end with inter-carrier-interference (ICI) also. Channel estimation also becomes complicated due to ISI as timing offset shifts channel impulse response.

Frequency mismatch between two oscillators at transmitter and receiver forms ICI at receiver. ICI disturbs fundamental requirement of OFDM link that is preservation of orthogonality among subcarriers. Thus ICI due to timing and frequency offset is a main barrier while setting up any OFDM based communication link. OFDM is very popular for its high data speed feature and that is the reason for its acceptance in all multimedia applications. There are many methods to handle synchronization issue. Pilot symbols based coarse timing and frequency acquisition and cyclic extension of OFDM symbol for fine frequency and timing synchronization are proposed by many authors. Over and above, it is also must to have common algorithm for handling the issue of synchronization because of rapid increase of mobile phone use comprising all types of communication in last decade. In short, proper and effective synchronization algorithms have been investigated for enhancing the performance of the OFDM system against timing and frequency errors.

Wireless Local Area Network (WLAN) standard 802.11 also use OFDM link which is really a great walk in order to remove wires from offices and laboratories. This paper focuses on theory and implementation of synchronization algorithms in 802.11ac WLAN standard. Long Term Evolution (LTE) - 4G employs pilot subcarriers instead of preamble symbols to set up easy process to minimize the timing and frequency synchronization errors. Similarly, DVB $-\mathrm{T} / \mathrm{H}$ also utilize dedicated subcarriers for timing and frequency synchronization purposes.

In practice, timing synchronization error is cancelled before handling the carrier frequency synchronization error. Generally, timing synchronization is performed in two parts: Rough timing synchronization and fine timing synchronization. Use of Cyclic prefix/suffix and dedicated preamble is the way to perform coarse timing synchronization. It is reviewed that the maximum likelihood (ML) [9], minimum mean squared error (MMSE) [10] and the maximum correlation (MC) methods [11] are three basic methods to resolve the problem of timing synchronization.

Cross correlation and Auto correlation based algorithms are normally used. Auto correlation based algorithms are easy to implement but they suffer with the plateau problem which occurs due to long repetition structure of training symbols. Cross correlation based algorithms solve this plateau problem at the cost of computational complexity and pseudo multipath problem due to delay introduced by multipath channel. Plateau and pseudo multipath problem due to auto correlation and cross correlation respectively were presented by many authors and proposed effective algorithms. Three step timing synchronization scheme having sliding window differentiator, SIR metric formation and frame timing refinement process [12] solved these problems well. Narae Kim proposed antenna selection algorithm [13] which reduces the power requirement of this three step method. It is clear that synchronization error is tackled at the cost of one of the parameters so adaptive use of cyclic prefix or training sequence will definitely overcome the synchronization error in real sense without much tradeoff.

Frequency synchronization error arises due to many reasons such as temperature changes, oscillator frequency drift due to doppler spread effect, tolerance of RF components etc. Carrier frequency offset (CFO) builds spreading of spectrum in the channel which finally turns out to be situation of orthogonality loss at the receiver [14]. This is called inter-carrier-interference. It is obvious that closed subcarriers are influenced by greater level of interference. CFO also attenuates received signal amplitude considerably. Detection method at receiver plays key role in the mitigation of $\mathrm{CFO}$ because it is observed that coherent detection is less influenced by $\mathrm{CFO}$ than non coherent detection. CFO if not approximated properly, it also deteriorates bit error rate (BER) in any channel.

CFO is also lowered down in steps like timing synchronization, means first rough approximation and after that fine tuning is done. It is certainly essential to estimate frequency error precisely to the half carrier spacing for reliable fine tuning process. It is experienced that coarse frequency synchronization with strict limitations gives escape from fine tuning process. Generally, coarse frequency synchronization means frequency acquisition algorithms provide sufficient accuracy. There are different algorithms exist to guess frequency errors. Pilot tone surrounded by zero valued virtual subcarriers, repeated data symbols and cyclic extension of 
OFDM symbols are very popular algorithms for CFO offset estimation. But these methods are using complex ML estimator does not fit with fast time varying channels. Complexity of ML estimator is minimized in the outstanding approach for estimating CFO by analyzing the fading process statistically. There are few algorithms suggesting CFO offset recovery in the frequency domain.

Obviously, CFO recovery is also application wise different for acceptable outcome so it is essential to compare different synchronization methods for designing common wireless device. Common device which can handle CFO in IEEE standards of Wireless LAN, 4G mobile communication and DVB-T/H is also suggested and proposed. [15].

\section{802.11ac FRAME}

Wired Local Area Network is now going to become past and it is being replaced by very trendy technology based on OFDM which is wireless and speedy. Because of advancement in the WLANs, it becomes popular at homes, offices and even public places. Since inception of IEEE 802.11 standard for WLAN, it has received remarkable response and shown great success. It is long journey of WLANs started from internet browsing, email services and moderate speed applications to high speed services include video streaming \& calling and transfer of large amounts of data. It is fact that no smart mobile device or tablet is available without built in WLAN card. It is high demand to provide high speed internet connectivity to the laptop and mobile phone which force research groups to throw updated standards like $802.11 \mathrm{ac}$ and 802.11 ad operating on the $5 \mathrm{GHz}$ band and the $60 \mathrm{GHz}$ band respectively. The 802.11ac is the extension of the successful standard 802.11n standard. It is also known as Very High Throughput (VHT). The 802.11ac is expanded in modulations up to 256 - QAM, bandwidth to $160 \mathrm{MHz}$ and up to 8 spatial streams for making it able to deliver data at the rate of 1 Gbps theoretically.

Many standards and amendments for WLANs have been released around basic standard 802.11a since 1999 and most of them in use today. First standard 802.11a was released in 1999 operating in the $5 \mathrm{GHz}$ with capability to reach a total throughput of $54 \mathrm{Mbps}$. Just after one year, in early 2000, IEEE launched $802.11 \mathrm{~b}$ operating in the $2.4 \mathrm{GHz}$ band with data rate of $11 \mathrm{Mbps}$ has come in the market. Subsequently, in 2001, $802.11 \mathrm{~b}$ was extended to new standard $802.11 \mathrm{~g}$ for increasing data rate up to $54 \mathrm{Mbps}$. Later in 2009, data rate was drastically uplifted to $600 \mathrm{Mbps}$ by amending standard 802.11n which is based on Multiple Input Multiple Output (MIMO) technique.

Next part of the paper describes frame structure of 802.11ac WLAN followed by synchronization algorithms implementation and results. The 802.11ac is the extension of 802.11a and 802.11n standards so that it reuses most of the part of these standards. 802.11ac is designed for the bandwidth of 20,40, $80 \mathrm{MHz}$ and optional 160 $\mathrm{MHz}$ to support data rate at least $1 \mathrm{Gbps}$ theoretically [16]. The subcarriers split from available bandwidth are separated by $312.4 \mathrm{KHz}$ spacing in 802.11ac standard. Synchronization errors are solved by pilot or training sequences which are carried by few subcarriers. These special designed training sequences are used as reference to estimate timing and frequency offset. Majority subcarriers are carrying data symbols. OFDM data symbol are suffixed or prefixed by sufficient guard interval to provide resistance to ISI which finally helpful to solve the problem of timing offset. But it increases payload to the data frame. This is called cyclic prefix or guard interval (GI). The GI is normally $20 \%$ of the OFDM symbol. Considering the symbol duration of $4 \mu \mathrm{s}$ and cyclic prefix $800 \mathrm{~ns}$ ( $20 \%$ of symbol duration) in IEEE 802.11 standards, total duration would be of $4.8 \mu \mathrm{s}$. It would allow receiver to handle small symbol timing inaccuracy due to delay spread of $600 \mathrm{~ns}$.

802.11ac data frame is also called Very High Throughput (VHT) frame. As seen in figure 1, it is understood in three parts; legacy preamble, VHT preamble and DATA. The Legacy preamble is used to offer support to the old versions of 802.11 standards. It is completed with three fields; Legacy Short Training Field (L-STF), a Legacy Long Training Field (L-LTF), and a Legacy Signal (L-SIG) field, same as used in 802.11a and 802.11n standards. These fields are followed by VHT preamble. These fields are main part of 802.11ac which support very high throughput. There are four fields which are VHT Signal-A (VHT-SIG-A), VHT Short Training (VHT-STF), VHT Long Training (VHT-LTF) and VHT Signal-B (VHT-SIG-B). frame ends with DATA symbols. 


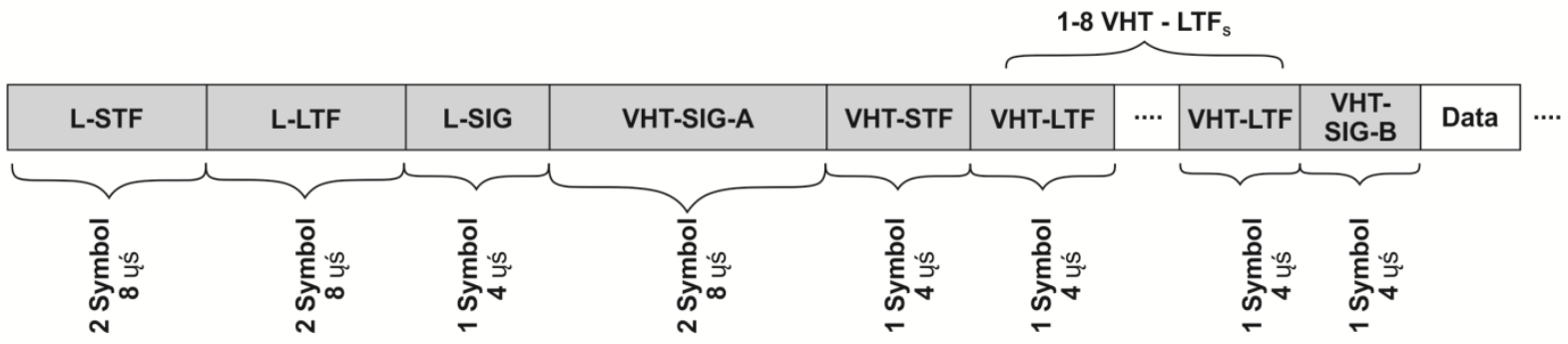

Fig.1. 802.11ac data frame

Legacy preamble is very important in 802.11ac frame to support previous standards. For $20 \mathrm{MHz}$ bandwidth, 12 subcarriers are being used for carrying a Legacy Short Training Field L-STF. It looks for time and frequency synchronizations as well as automatic gain control (AGC). Legacy Long Training Field is being carried by 52 subcarriers in $20 \mathrm{MHz}$ bandwidth which is especially important for channel estimation. L -SIG is the last field in legacy preamble which specifies data rate and length of packet as shown in figure 2 .

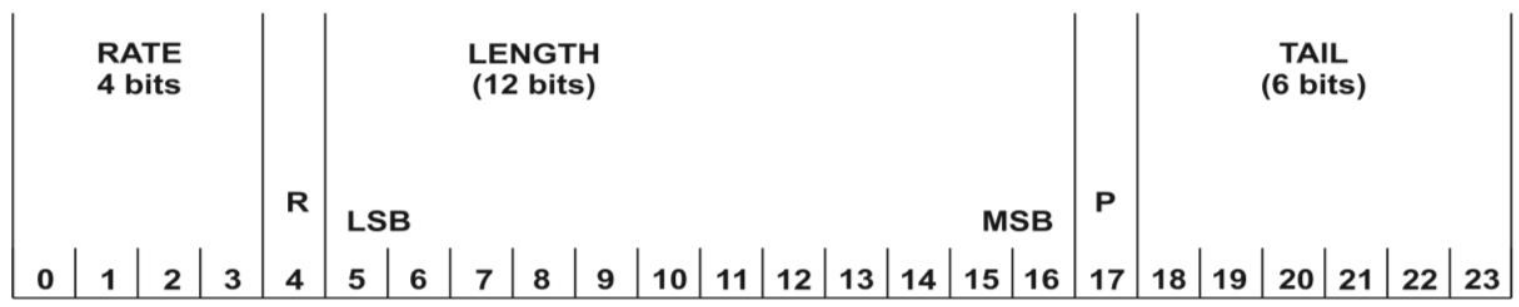

Fig.2. L-SIG Field

These fields are used by only 802.11ac standard and previous standards 802.11a and 802.11n ignore them. The VHT-SIG-A field consists of two symbols each having 24 bits. The first symbol VHT-SIG-A1 is Binary Phase Shift Keying (BPSK) modulated with binary convolution coding of rate $1 / 2$ and VHT-SIG-A2 is Quadrature BPSK modulated. They depict channel bandwidth, coding and whether frame is for single user or multi user. It also contains field to indicate whether a short GI is used or not, type of encoding, beam forming as shown in figure 3 .

After coarse timing estimation, fine estimation is performed using field VHT-STF. This field is also provides information for controlling gain automatically MIMO OFDM transmission. This field is similar to L-STF and carries a small portion of the subcarriers, while remaining subcarriers are all set to zero. Channel estimation and equalization is being done in MIMO receiver with the help of VHT-LTF. One symbol of VHT-SIG-B field with Bipolar Phase Shift Keying modulation indicates the length of the transmitted data. Figure 4 shows fields of VHT-SIG-B for single user and VHT-SIG-B for different bandwidths. 


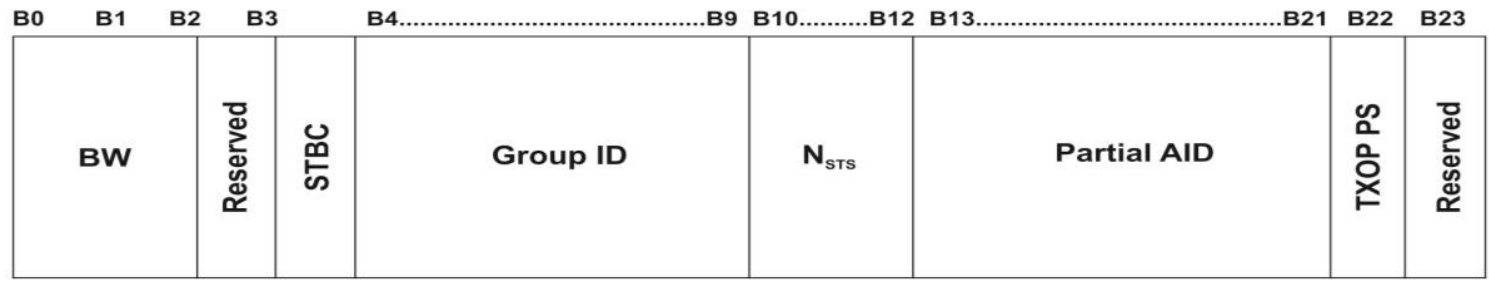

VHT-SIG-A,

\begin{tabular}{|c|c|c|c|c|c|c|}
\hline B0 B1 & B3 & 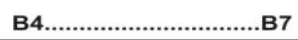 & B8 & B9 & B10................................. & В18.................23 \\
\hline Short GI & Coding & MCS & 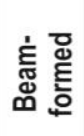 & 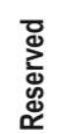 & CRC & Tail Bits \\
\hline
\end{tabular}

VHT-SIG-A

Fig.3. VHT-SIG-A Field

\begin{tabular}{|l|c|c|}
\hline B0 ….............................. B16,18,20 & $+2,3$ bits & B20,21,23 ............ B225,26,28 \\
\hline Length & Reserved & Tail Bits \\
\hline
\end{tabular}

Single-User VHT-SIG-B

$20 \mathrm{MHz}$

\begin{tabular}{|c|c|}
\hline 20 bits & $\begin{array}{c}6 \text { Tail } \\
\text { Bits }\end{array}$ \\
\hline
\end{tabular}

$40 \mathrm{MHz}$

\begin{tabular}{|c|c|c|c|}
\hline 21 bits & $\begin{array}{c}6 \text { Tail } \\
\text { Bits }\end{array}$ & 21 bits & $\begin{array}{c}6 \text { Tail } \\
\text { Bits }\end{array}$ \\
\hline
\end{tabular}

$80 \mathrm{MHz}$

\begin{tabular}{|c|c|c|c|c|c|c|c|c|}
\hline 23 bits & $\begin{array}{c}6 \text { Tail } \\
\text { Bits }\end{array}$ & 23 bits & $\begin{array}{c}6 \text { Tail } \\
\text { Bits }\end{array}$ & 23 bits & $\begin{array}{c}6 \text { Tail } \\
\text { Bits }\end{array}$ & 23 bits & $\begin{array}{c}6 \text { Tail } \\
\text { Bits }\end{array}$ & $\begin{array}{c}\text { Pad } \\
\text { Bit }\end{array}$ \\
\hline
\end{tabular}

$160 \mathrm{MHz}$

x 2

Fig. 4 VHT-SIG-B Field 
The last field in the 802.11ac frame is DATA field. It consists SERVICE field and Physical layer Service Data Unit (PSDU) followed by optional padding and tail bits. Number of OFDM symbols in PSDU to be transmitted is variable which is set in L-SIG field. Service field also carries information about scrambler initialization.

\section{Synchronization Algorithms \& Results}

This section highlights timing and frequency synchronization algorithms for 802.11ac and briefs the results. Equation 1 represents the effect of timing and frequency offset in the signal at receiver. This is very complicated situation for any algorithm to distinguish between phase shifts introduced by timing and frequency offset [17].

$$
S_{n, i}=\left(\frac{1}{\sqrt{N}} \sum_{k=0}^{N-1} \mathrm{~S}_{\mathrm{n}, \mathrm{k}} H_{K} e^{j \frac{2 \pi k i}{N}} . e^{j \frac{2 \pi k \delta i}{N}} \cdot e^{j 2 \pi(i+\delta i) \frac{\delta k}{N}}\right)+w_{n, i+\delta i}
$$

Where

$\mathrm{S}_{\mathrm{n}, \mathrm{k}}=$ OFDM data symbol of $\mathrm{n}^{\text {th }}$ frame on $\mathrm{k}^{\text {th }}$ subcarrier

$\mathrm{i}=$ Number of discrete time slot

$\mathrm{N}=$ Total number of subcarriers

$\mathrm{H}_{\mathrm{k}}=$ Channel response at each subcarrier

$e^{j 2 \pi(i+\delta i) \frac{\delta \kappa}{N}}=$ phase shift due to frequency offset $\delta_{k}$

$e^{j \frac{2 \pi k \delta i}{N}}=$ phase shift due to timing offset $\delta_{i}$

As discussed earlier, frame alignment against timing offset should be attained before estimating the frequency offset and correction.

Short training sequence consists of 10 repeated symbols used for time synchronization and coarse frequency estimation while the fine frequency estimation is done by long training sequence of 2 repeated symbols. Repeated structure of the 10 short symbols is used to track the arrival of signal by computing mean square error (MSE) between two short symbols. Figure 5 shows the detection of signal using 10 short symbols when signal is delayed by 500 samples and 1000 samples.

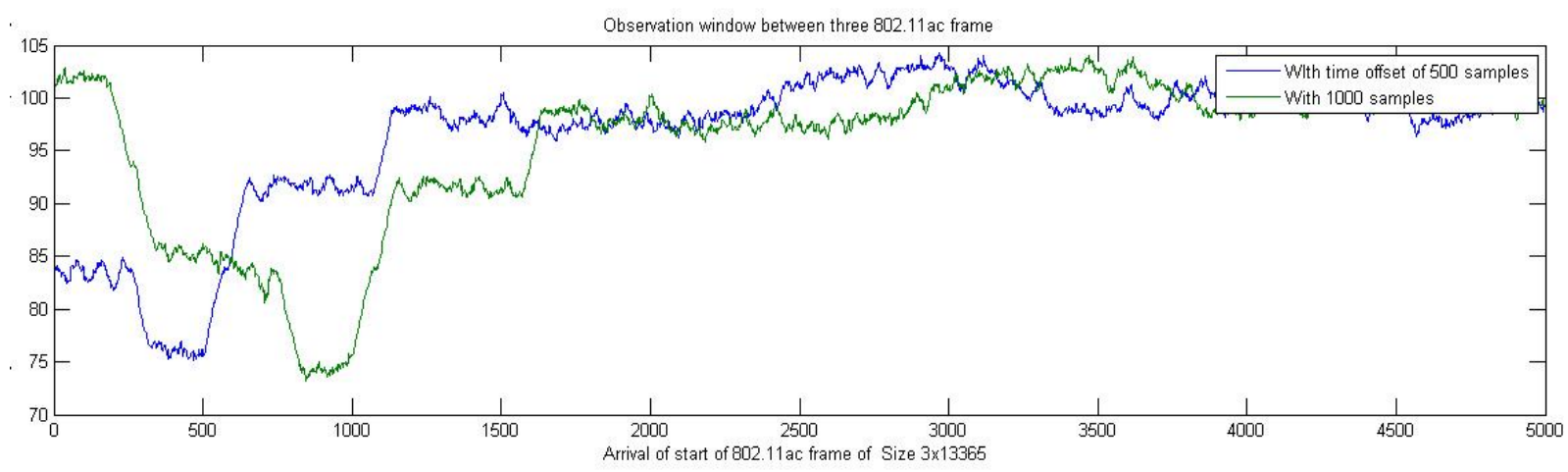

Fig.5. Detection of time arrival of short sequence 
After detection and estimation offset, it is very easy to compensate it for better synchronization as it has shown in following figure 6.

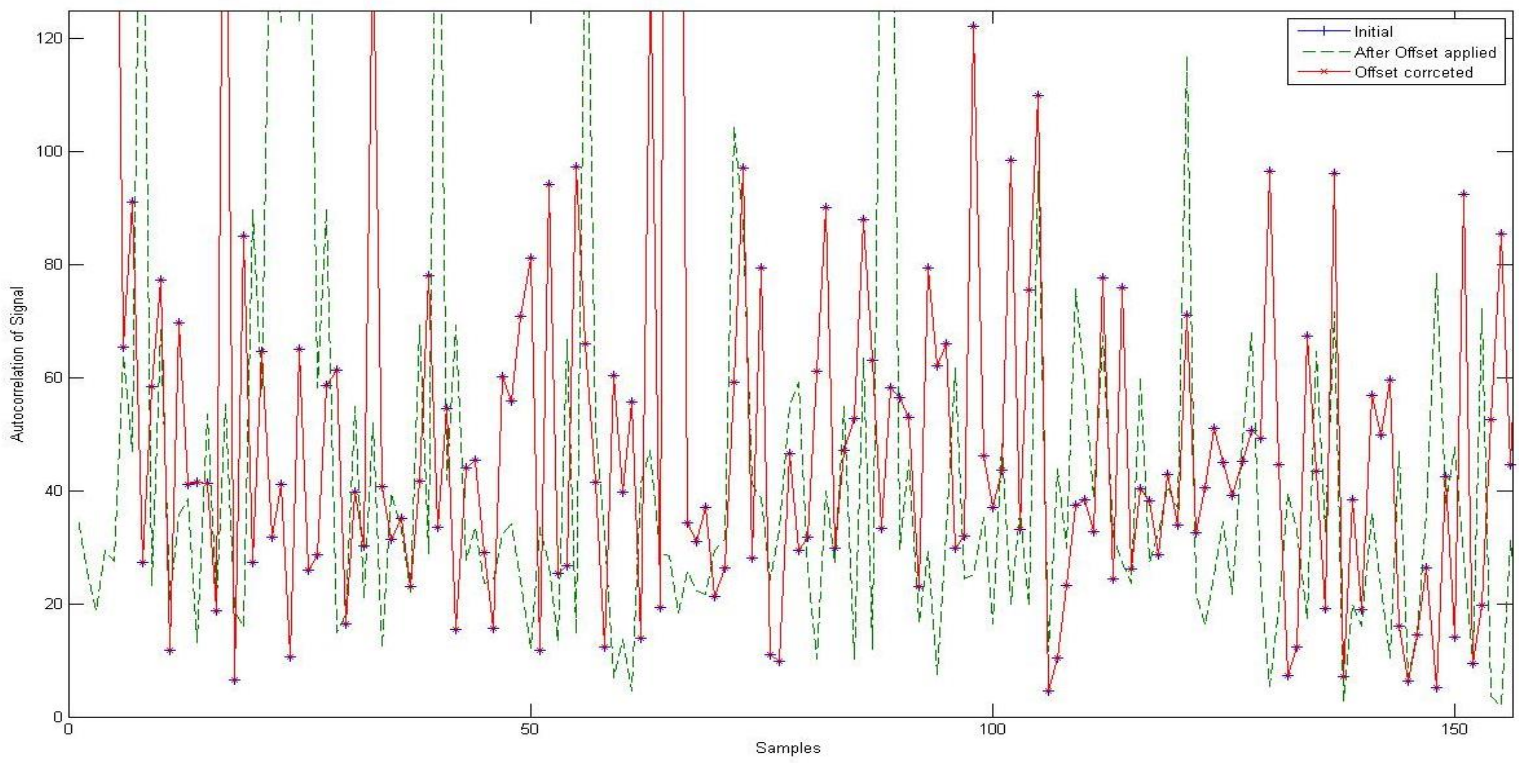

Fig.6. OFDM Signal: after \& before time offset

Now it is good to estimate frequency offset roughly using 10 short symbols first and then fine estimation using 2 long symbols. Correlation between adjacent short symbols is used to estimate the frequency offset. There are three following methods implemented in 802.11ac receiver to estimate frequency offset. In method 1 , correlation between two adjacent symbols is computed as below.

$$
Z_{\frac{L}{2}}=\sum_{i=0}^{\frac{L}{2}-1} s_{n, i} \cdot s_{n, i+\frac{L}{2}}^{*}
$$

Where $N$ indicates FFT length and $L$ is the total length of two adjacent identical symbols. Argument of expected value of this correlation is calculated to finally determine carrier frequency offset.

$$
\delta \hat{k}=-\arg \left\{E\left[\hat{Z}_{\frac{L}{2}}\right]\right\} \cdot \frac{N}{\pi \cdot L}
$$

Maximum 9 such correlations can be formed from the 10 symbols so that in method 2, average of 9 correlations is taken to determine frequency offset.

$$
\delta \hat{k}_{10 \text { short } / 9 \text { corr }}=\frac{1}{9} \sum_{i=1}^{9} \delta \hat{k}_{2 \text { Short }, i}
$$


Where

$$
\hat{Z}_{\text {Short }}=\sum_{i=1}^{16} \hat{s}_{n, i}^{*} \cdot \hat{s}_{n, i+16} \& \delta \hat{k}_{2 \text { Short }}=-\arg \left\{\hat{Z}_{\text {Short }}\right\} \cdot \frac{N}{\pi \cdot L}
$$

In method 3, alternate symbols are taken in the computation of correlation so that total 5 correlations are computed and average of these is taken to estimate frequency offset.

$$
\hat{\delta K_{10 \text { short } / 5 c o r r}}=\frac{1}{5} \sum_{i=1}^{5} \delta \hat{K}_{2 \text { Short }, i}
$$

Where

$$
\hat{Z}_{\text {short }}=\sum_{i=1}^{16} \hat{S}_{n, i} \cdot \hat{S}_{n, i+16 * 5}
$$

Final stage of synchronization process is fine synchronization for frequency offset which is handled by correlation of two long symbols.

$$
\hat{\delta}_{2 \text { Long }}=-\arg \left\{\hat{Z}_{\text {Long }}\right\} \cdot \frac{N}{\pi \cdot L}
$$

Where

$$
\hat{Z}_{\text {Long }}=\sum_{i=1}^{64} \hat{S}_{n, i+32} \cdot \hat{S}_{n, i+32+64}
$$

These methods are implemented in 802.11ac standard of WLAN and results are analyzed. Equation 1 clearly indicates that OFDM signal at the receiver influenced by the phase shifts due to timing offset as well as frequency offset along with noise. After timing synchronization, coarse frequency estimation and correction is implemented using above mentioned three methods of correlation represented by equations $3,4, \& 5$. Figure 7 shows comparison of the three methods in which it is found that method 3 of 5 correlations average gives better result compared to other two. At $10 \mathrm{~dB}$, BER is about 0.125 for method 3 while for method 1 and 2 , it is close to 2.0 . 


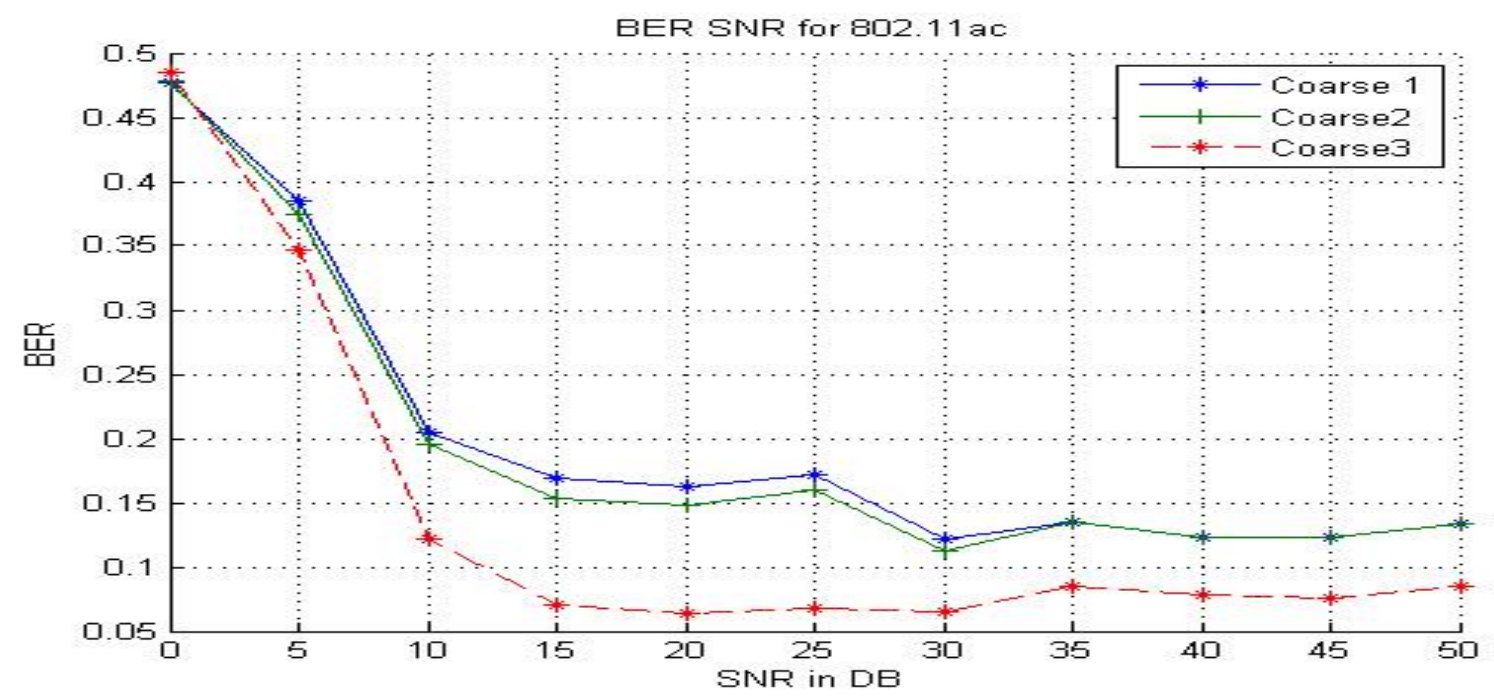

Fig.7. Comparison: coarse estimation of frequency offset

Later, as per equation 6, frequency is finely estimated and observed that it has improved BER a lot in method 3. Fine estimation in method 3 reduces BER from 0.125 to 0.07 , almost 50 percent. There is also improvement in the BER for method 1 and method 2. Figure 8 shows variations in BER after fine estimation of frequency offset implementation for all three methods. It is also observed that method 3 is best at all values of SNR and shows noticeable and remarkable difference in the improvement of BER. Drastic reduction in BER is the extra benefits of method 3 which gives reasonable quality even in worst situation of channel.

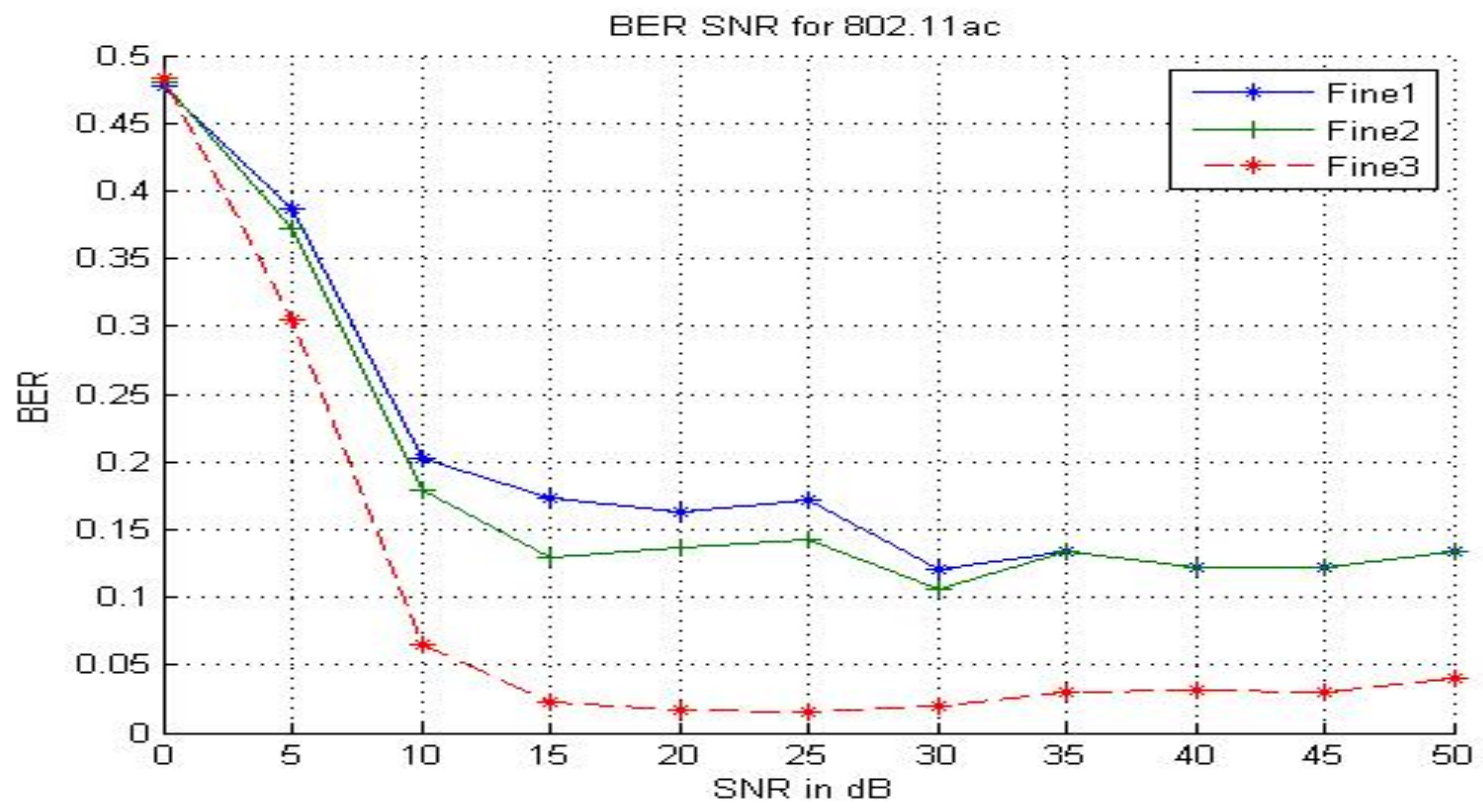

Fig.8. Comparison: fine estimation of frequency offset 
Effect of CFO in different methods is also tested. Variation of BER with reference to CFO is also seen for different value of SNR in figure 9. For higher value of SNR, performance improvement is achieved. Following graph shows BER variation with respect to CFO for SNR value $10 \mathrm{~dB}$.

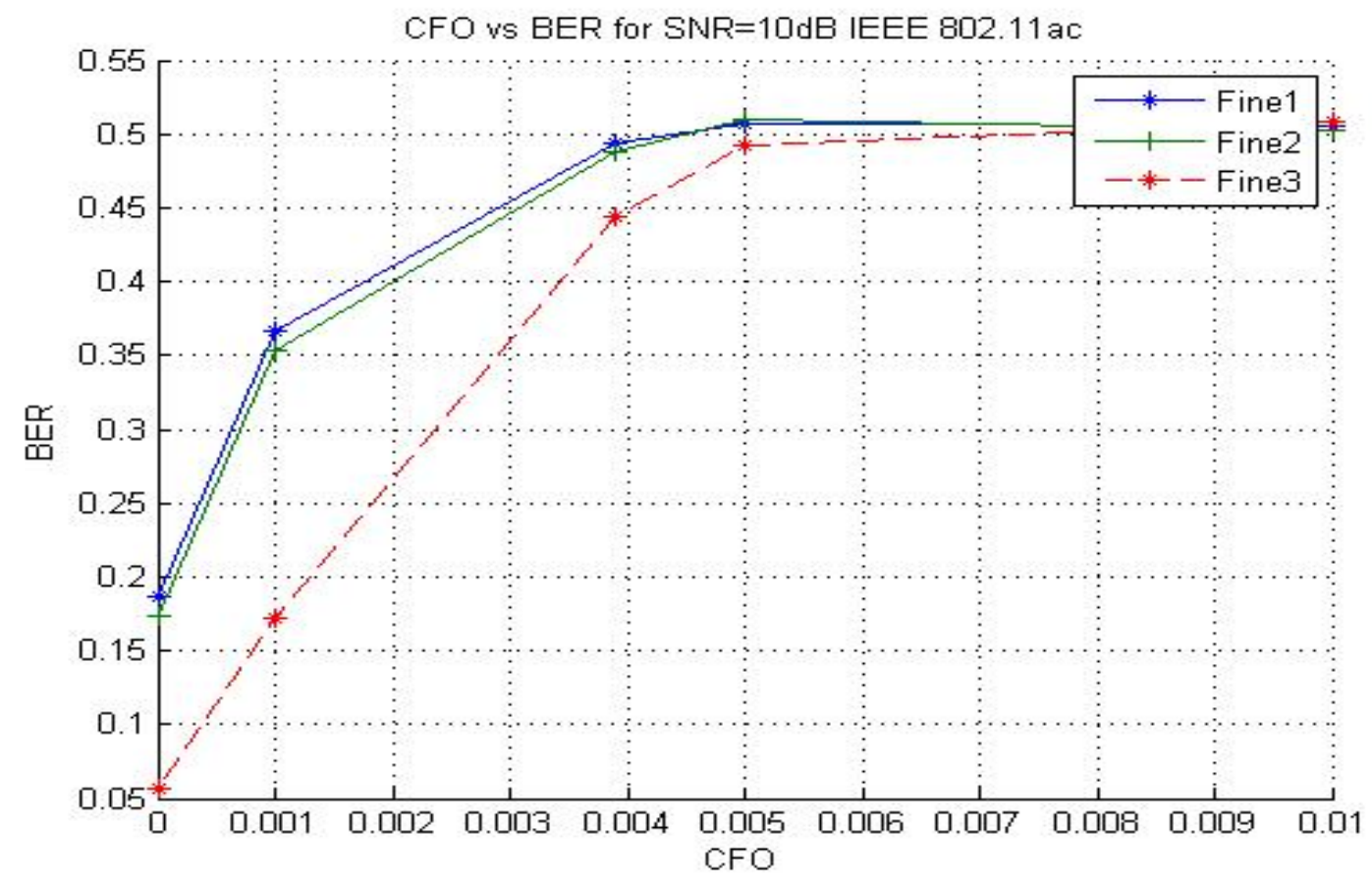

Fig.9. BER V/s. CFO for SNR $10 \mathrm{~dB}$.

It is observed that CFO variation between 0.001 to 0.005 introduces large variation in BER for the method 1 and method 2 compared to method 3. It also reveals that the effect of methods becomes ineffective after a particular value of CFO.

\section{Conclusions}

We have touched and tried to elaborate very important issue of OFDM system which is being used in most of the high speed communication links. There is no straight process to be implemented for the improvement of BER against synchronization issue. We have surveyed various methods of synchronization and few of them pointed out in this paper. It is indeed an always open area to get better and better outcomes because OFDM modulation technique has been used in many applications and will be used in upcoming applications with or without modifications. It would be acceptable to use various methods to in switching to attain targeted BER against ISI and ICI with less complexity. Adaptive synchronization methods would be preferred and accepted in the future. Complexity in system and overloading in data structure will be reduced in adaptive synchronization. Common device for different applications requirement will be challenge for the designers as all applications have different data frame structure. 


\section{References}

[1] ETSI EN 300 744, "Digital video broadcasting (DVB): frame structure, channel coding and modulation for digital terrestrial television (DVB-T)," Tech. Rep., ETSI, 2004

[2] "IEEE draft standard for IT - telecommunications and information exchange between systems LAN/MAN - specific requirements - part 11: Wireless LAN medium access control and physical layer specifications - amd 4: Enhancements for very high throughput for operation in bands below 6GHz," IEEE P802.11ac TM/D3.0, pp. 1-385, June 2012.

[3] "IEEE standard for local and metropolitan area networks part 16: air interface for fixed broadband wireless access systems," IEEE 802.16, 2004.

[4] A. B. Ericsson, "Long term evolution (LTE): an introduction," White paper, October 2007.

[5] Chandresh D. Parekha, Jayesh M. Patel, "Overview on Synchronization in OFDM Systems" in IEEE International Conference on Advances in Computing, Communication and Automation ICACCA-2016, (Spring) ISBN 978-1-5090-0673-1, April, 2016.

[6] Zhou, Hao, Amaresh V. Malipatil, and Yih-Fang Huang. "Synchronization issues in OFDM Systems." Circuits and Systems, 2006. APCCAS 2006. IEEE Asia Pacific Conference on. IEEE, 2006.

[7] K.Wang, M.Faulkner, J. Singh and I.Tolochko, "Timing synchronization for 802.11a WLANs under multipath channels," in ATNAC, Melbourne, Dec 2003 http://atnac2003.atcrc.com/FinalProgram.html.

[8] S Nandula, K Giridhar, "Robust timing synchronization for OFDM based wireless LAN System," Proc. IEEE International Conference on Convergent Technologies for Asia-Pacific Region, TENCON 2003, vol 4C, pp. 1558-1561, Bangalore, India, 2003.

[9] J. J. van de Beek, M. Sandell, and P. O. Borjesson, "ML estimation of timing and frequency offset in OFDM systems," in IEEE Trans. Signal Processing, vol. 45, no. 6, pp. 1800 - 1805, July. 1997.

[10] M. Speth, F. Classen, and H. Meyr, "Frame synchronization of OFDM systems in frequency selective fading channels," in Proc. VTC, pp. 1807-1811, 1997.

[11] M. Speth, S. Fechtel, G. Fock, and H. Meyr, "Optimum Receiver Design for OFDM-Based Broadband Transmission - Part II: A Case Study," in IEEE Trans. on Comm., vol. 49, no. 4, pp. 571 - 578, April. 2001.

[12] Dong Wang; Jinyun Zhang, "Timing Synchronization for MIMO-OFDM WLAN Systems," in Wireless Communications and Networking Conference, 2007.WCNC 2007. IEEE , vol., no., pp.1177-1182, 11-15 March 2007 doi: 10.1109/WCNC.2007.223

[13] Narae Kim, Suhyun Kim, Yunho Jung, and Seongjoo Lee, “A low power antenna selective synchronization method for IEEE 802.11ac WLAN Systems," in Journal of Electromagnetic Waves and Applications, 12 May, 2015 http://dx.doi.org/10.1080/09205071.2015.1035865

[14] Ma, X.; Kobayashi, H.; Schwartz, S.C, "Effect of frequency offset on BER of OFDM and single carrier systems," in IEEE International Conference on Communications, vol. 3, pp. 22-27, June 1996.

[15] J. González-Bayón, C. Carreras and O. Edfors, "A Multistandard Frequency Offset Synchronization Scheme for 802.11n, 802.16d, LTE, and DVB-T/H Systems", Journal of Computer Systems, Networks, and Communications, vol. 2010, pp. 1-9, 2010.

[16] Al-Ghazu, Nader. A Study of the Next WLAN Standard IEEE 802.11 ac Physical Layer. Stockholm: Sweden, 2013.

[17] Lui, Cheuk Kwan. method of synchronization using IEEE 802.11 a OFDM training structure for indoor wireless applications. Diss. School of Engineering Science-Simon Fraser University, 2004. 


\section{Authors' Profiles}

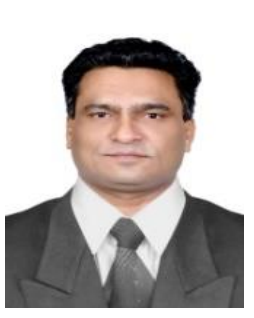

Chandresh Parekha (born October 21, 1972) is an academician for more than 15 years and currently serving as an Assistant Professor in Telecommunication at Raksha Shakti University, Ahmedabad, Gujarat, India. He completed B.E. (Electronics) in 1994 and M.E. (Electronics Communication System) in 2009. He also worked as an R \& D engineer in Crown Television Limited, Gandhinagar, Gujarat, India and Videocon International Limited, Gandhinagar, Gujarat, India. He has been actively involved in academics by publishing more than 50 research papers in reputed journals/conferences, doing various funded research projects, delivering expert talks and organizing technical events.

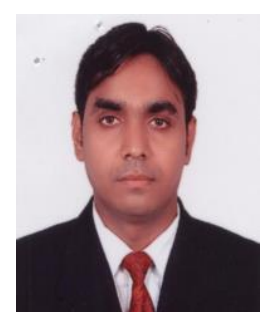

Dr. Jayeshkumar Madhubhai Patel received the $\mathrm{PhD}$ degree in Computer Science from the North Gujarat University, Gujarat, India, in 2006. In 2003, Dr. Patel became Ph.D. Guide from last 12 years. Since 2011, he has been at the Ganpat University; where he is presently an Associate Professor of Artificial Intelligence included teaching in Post Graduate Subjects in the M.C.A. Department. His current research interests include knowledge representation systems, integration of artificial intelligence techniques with neural networks, text classification, pattern recognition, and document processing, in particular. He is a member of the CSTA, IAENG. Dr. Patel has been responsible for several projects and has published more than 64 papers in international conference proceedings and scientific journals.

How to cite this paper: Chandresh D. Parekha, Jayesh M. Patel, " OFDM Synchronization Techniques for 802.11ac WLAN", International Journal of Wireless and Microwave Technologies(IJWMT), Vol.8, No.4, pp. 1-13, 2018.DOI: 10.5815/ijwmt.2018.04.01 